\title{
A simple coupled mode model for near band-edge phenomena in grated waveguides
}

\author{
H.J.W.M. Hoekstra, W.C.L. Hopman, J. Kautz, R. Dekker and R.M. de Ridder \\ Integrated Optics Micro Systems group, University of Twente, P.o. box 217, 7500 AE Enschede, The \\ Netherlands, \\ h.j.w.m.hoekstra@ewi.utwente.nl
}

The paper presents a simple coupled mode model to describe near band-edge phenomena in the transmission spectra of waveguides with a section containing a rectangular grating.

\section{Summary}

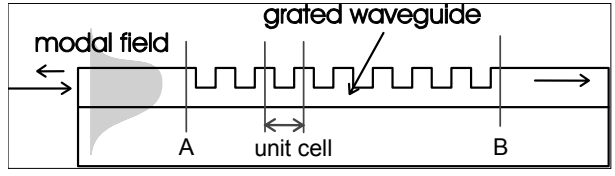

Figure 1. Sketch of a waveguide with a grated section; $A$ and $B$ are reference planes for defining the grated section.
It is well known that the features of the near band-edge transmission spectrum of a waveguide with a partly grated section (see figure 1) are dominated by the effects of strong dispersion. In this wavelength region sharp transmission peaks (see figure 2) are found corresponding to strong field enhancement inside the cavity, defined by the grated section. These phenomena can be explained by considering the grating section as a

Fabry-Perot cavity for the grating mode(s) (strong reflection at the boundaries of the grating section), together with the dispersive properties of these modes which are composed of forward and backward moving fields.

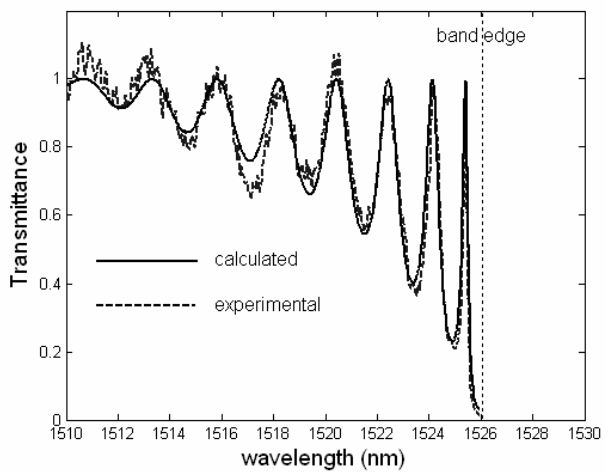

Figure 2. Experimental transmittance of a grated waveguide and results calculated with the presented model
It is the aim of the paper to show that from the wavelength position of the near band-edge transmission peaks most of the relevant parameters of the grating device can be deduced. First it is argued that, due to the symmetry of the grating (with rectangular grooves) the modal reflection and transmission coefficients for the transitions are nearly real. Using the latter, the approximate shape of the near bandedge dispersion curve, defined by $\beta(\lambda)$ with $\beta$ the propagation constant and $\lambda$ the wavelength, of the propagating grating modes can be deduced from the positions of the transmission peaks for a structure with a given grating length. Next, from the dispersion curve most of the relevant

device parameters, such as width of the peaks, field enhancement, modal group velocity, group delay and peak position as a function of the length of the grated section can be derived in good approximation. The presented theory is illustrated with numerical calculations and results of experiments on waveguides with grated sections. 